Haemostasis 1996;26 (Suppl 4):391-392

\title{
Subjects Index
}

Adenovirus, 132

Angioplasty, 285

Animals models, 55

Anticardiolipin antibodies, 55

Anticoagulant therapy, 55, 220, 248, 266

Antiphospholipid syndrom, 389

Anhphospholipids antibodies, 339, 357

Antiplatelet agent, 55

Antiprothrombin antibody, 339

Antiß2 glycoprotein, 1, 339, 357

Antithrombotic strategies, 269

Apoptosis, 37

Arachidonic acid, 28

Argatroban, 70

Arterial thrombosis, 55, 78, 227

Aspirin, 78

Atherothrombogenesis, 46

Athrosclerotic lesions, 269

Avian hemengioma, 37

ß2 glycoprotein, 1, 357 ß2 integrins, 20 Buflomedil, 182

Cardiovascular risk factor, 166 Cathepsin G, 20 Cell-cell interaction, 20 Circulation, 28

Coagulation inhibitor, 154 Coagulation system, 132 Cytokines, 330

D-Dimer, 1

Deep vein thrombosis, 220, 367 Dermatan sulphate, 367 DuP714, 70

Efegatran, 70

Endothelial cells, 28, 37, 177, 182, 210, 233

Enzyme kynetics, 98

Experimental thrombosis, 55

Fl+2, 1

Factor V Leiden, 300, 314

Factor X, 98

Factor $\mathrm{X}$ activation, 89, 98

Fatty acids, 46

Fetal los, 339, 357

Fibrinogen, 330

Fibrinolysis, 132

Flow reactor, 89

G-Proteins, 199 Gene therapy, 132 Glycoprotein polymorphism, 78

Heart, 28 
Hemorheology, 166, 171 Heparin, 154, 189,220 Hereditary defidency, 9 Hip arthroplasty, 378 Hiradin, 70, 367 Hirudisins, 70 Hirulog, 70, 367 Hypercholesterolemia, 166, 171

Hypercoagulable states, 1 Hypertension, 166 Hypoxia, 182 Hypoxia-reoxygenation, 177, 182 Infection, 233

Inflammation, 210, 233

Inhibition of GP lib Illia complexes, 78

Inogatran, 70

Integrin receptors, 285 Intercellular junctions, 210 International normalized ratio, 248, 266

Ischemia-reperfusion, 182

Leucocytes, 210

Leukotriène C4, 28

Lipid-rich plaques, 269

lipoproteins, 154

LMWH, 189,220,278

Lovastatine, 171

Lupus anticoagulant, 339, 357

Markers of coagulation activator, 1

Mutation, 9

Myocardial infarction, 227, 289

Napsatroban, 70 Neutrophil adhesion, 182 Neutrophils, 28

Oral anticoagulants, 248

Orgaran, 367

Out of hospital treatment, 189

P selectin: 20, 177, 182

Palm oil, 46

Pentasacharide, 367

Phospholipids, 89, 98

Plasma lipoproteins, 46

Plasminogen system, 132

Platelet activation, 102, 199

Platelets, 20, 78, 102

Platelets GPIlb/lla, 285

PMN leucocytes, 20

Post operative thromboembolism, 378

Pregnancy, 244

Prolonged prophylaxis, 378

Protein C, 9

Prothrombin time, 248

Retrovirus, 37

Risk factor of thrombosis, 78, 269, 330

Signal transduction, 199 Staphylokinase, 293

Thrombolytic Therapy, 293

Thrombomodulin, 227

Thrombophilia, 1, 300, 314

Thromboresistance, 37

Thrombosis, 269, 285, 339, 357 
Tlclopidine, 78

Tissue factor, 89, 98, 154

Tissue factor pathway inhibitor, 89, 154,

Transgenic mice, 132

Treatment of venous thrombosis, 189

Unfractionated heparin, 189 Unstable angina, 285

Venous Thrombosis, 227, 300, 314 Vessel wall, 28

Warfarin, 220

Randomised trials, 220

Red blood cell membrane lipids, 171

Receptors, 199

Resistance to activated protein C, 300, 314 